\title{
RATIONALLY ISOTROPIC QUADRATIC SPACES ARE LOCALLY ISOTROPIC. III
}

\author{
I. PANIN AND K. PIMENOV
}

Dedicated to Professor S. V. Vostokov

with great respect

\begin{abstract}
Let $R$ be a regular semilocal domain containing a field such that all the residue fields are infinite. Let $K$ be the fraction field of $R$. Let $\left(R^{n}, q: R^{n} \rightarrow R\right)$ be a quadratic space over $R$ such that the quadric $\{q=0\}$ is smooth over $R$. If the quadratic space $\left(R^{n}, q: R^{n} \rightarrow R\right)$ over $R$ is isotropic over $K$, then there is a unimodular vector $v \in R^{n}$ such that $q(v)=0$. If $\operatorname{char}(R)=2$, then in the case of even $n$ the assumption on $q$ is equivalent to the fact that $q$ is a nonsingular quadratic space and in the case of odd $n>2$ this assumption on $q$ is equivalent to the fact that $q$ is a semiregular quadratic space.
\end{abstract}

\section{$\S 1$. INTRODUCTION}

This paper extends the main results of $[\mathrm{Pa}, \mathrm{PaP}]$ to the case of characteristic two. The new proof relies upon a new idea not involving resolution of singularities in any form.

Let $k$ be an infinite field, possibly with $\operatorname{char}(k)=2$, let $X$ be a $k$-smooth irreducible affine scheme, and let $x_{1}, x_{2}, \ldots, x_{s} \in X$ be closed points. Let $P$ be a free $k[X]$-module of rank $n>0$. If $n$ is odd, then let $(P, q: P \rightarrow k[X])$ be a semiregular quadratic module over $k[X]$ in the sense of $[\mathrm{Kn}$, Chapter IV, §3]. If $n$ is even, then let $(P, q: P \rightarrow k[X])$ be a quadratic space in the sense of $[\mathrm{Kn}$, Chapter I, (5.3.5)].

Remark 1. In both cases, this is equivalent to saying that the $X$-scheme $Q:=\{q=0\} \subset$ $\mathbf{P}_{X}^{n-1}$ is smooth over $X$.

Proof. For quadratic forms over a field, being regular or semiregular in the sense of $\mathrm{Kn}$, Ch. I, (5.3.5)] is equivalent to being nondegenerate in the sense of [EKM, $\S 7 \mathrm{~A}]$. In this case, smoothness is equivalent to nondegeneracy by [EKM, Proposition 22.1]. To extend the assertion to quadratic forms over rings, one can use [Kn, Chapter IV, (3.1.5)].

Let $p: Q \rightarrow X$ be the projection. For a nonzero element $f \in k[X]$, let $Q_{f}=p^{-1}\left(X_{f}\right)$, where $X_{f}$ is the principal open subset. Let $U=\operatorname{Spec}\left(\mathcal{O}_{X,\left\{x_{1}, x_{2}, \ldots, x_{s}\right\}}\right)$. Set ${ }_{U} Q=U \times_{X} Q$. For a $k$-scheme $D$ equipped with $k$-morphisms $U \leftarrow D$ and $D \rightarrow X_{f}$, set ${ }_{D} Q={ }_{U} Q \times{ }_{U} D$ and $Q_{D, f}=D \times X_{f} Q_{f}$.

Proposition 2. If $n>1$, then there exists a finite surjective étale $k$-morphism $U \leftarrow D$ of odd degree, a morphism $D \rightarrow X_{f}$, and an isomorphism of D-schemes ${ }_{D} Q \stackrel{\bar{\Phi}_{D}}{\longleftarrow} Q_{D, f}$.

2010 Mathematics Subject Classification. Primary $13 \mathrm{H} 05$.

Key words and phrases. Quadratic form, regular local ring, isotropic vector, Grothendieck-Serre conjecture.

Theorem 3 was proved with the support of the Russian Science Foundation (grant no. 14-11-00456). The research of the second author was partially supported by RFBR grant 12-01-33057 "Motivic homotopic cohomology theories on algebraic varieties" and by RFBR grant 13-01-00429 "Cohomological, classical, and motivic approach to algebraic numbers and algebraic varieties". 
Given this proposition, we may prove the following theorem.

Theorem 3. Assume that $f \in k[X]$ is a nonzero element such that there is a section $s: X_{f} \rightarrow Q_{f}$ of the projection $Q_{f} \rightarrow X_{f}$. Then there is a section $s_{U}: U \rightarrow{ }_{U} Q$ of the projection $U Q \rightarrow U$.

Proof of the Main Theorem. We give a proof of the theorem only in the local case and leave the semilocal case to the reader. So, $s=1$, and we shall write $x$ for $x_{1}$ and $\mathcal{O}_{X, x}$ for $\mathcal{O}_{X,\left\{x_{1}\right\}}$. If $f \in k[X]-m_{x}$, then there is nothing to prove. Now, let $f \in m_{x}$, then by Proposition 2 there is a finite surjective étale $k$-morphism $U \leftarrow D$ of odd degree, a morphism $D \rightarrow X_{f}$, and an isomorphism of the $D$-schemes ${ }_{D} Q \stackrel{\bar{\Phi}_{D}}{\longleftarrow} Q_{D, f}$.

The section $s$ defines a section $s_{D}=(i d, s): D \rightarrow Q_{D, f}$ of the projection $Q_{D, f} \rightarrow D$. Next, $\bar{\Phi}_{D} \circ s_{D}: D \rightarrow{ }_{D} Q$ is a section of the projection ${ }_{D} Q \rightarrow D$. Finally, if $p_{1}:{ }_{D} Q \rightarrow{ }_{U} Q$ is the projection, then $p_{1} \circ \bar{\Phi}_{D} \circ s_{D}: D \rightarrow U Q$ is a $U$-morphism of $U$-schemes. Recall that $U \leftarrow D$ is a finite surjective étale $k$-morphism of odd degree and $U$ is local with an infinite residue field. Therefore, by a version of Springer's theorem proved in $[\mathrm{PR}]$, there is a section $s_{U}: U \rightarrow{ }_{U} Q$ of the projection ${ }_{U} Q \rightarrow U$. (If $\operatorname{char}(k)=2$, the proof of the version of Springer's theorem given in $[\mathrm{PR}]$ works well with a very mild modification). The theorem is proved.

The Main Theorem has the following immediate consequences.

Corollary 4. Let $\mathcal{O}=\mathcal{O}_{X,\left\{x_{1}, x_{2}, \ldots, x_{s}\right\}}$ be a semilocal ring as above, and let $k(X)$ be the rational function field on $X$. Let $P$ be a free $\mathcal{O}$-module of rank $n>1$ and $q: P \rightarrow$ $\mathcal{O}$ a form over a ring $\mathcal{O}$ as above, that is, the $\mathcal{O}$-scheme $Q:=\{q=0\} \subset \mathbf{P}_{\mathcal{O}}^{n-1}$ is smooth over $\mathcal{O}$. If the equation $q=0$ has a nontrivial solution over $k(X)$, then it has a unimodular solution over $\mathcal{O}$.

Corollary 5. Let $R$ be a semilocal regular domain containing a field, and let $R$ be such that all the residue fields are infinite. Let $K$ be the fraction field of $R$. Let $P$ be a free $R$-module of rank $n>1$ and $q: P \rightarrow R$ a quadratic form over $R$ such that the $R$-scheme $Q:=\{q=0\} \subset \mathbf{P}_{R}^{n-1}$ is smooth over $R$. If the equation $q=0$ has a nontrivial solution over $K$, then it has a unimodular solution over $R$.

Corollary 6. Let $R$ be a semilocal regular domain containing a field, and let $R$ be such that all the residue fields are infinite. Let $K$ be the fraction field of $R$. Let $P$ be a free $R$-module of even rank $n>0$ and $q: P \rightarrow R$ a quadratic form over $R$ such that the $R$-scheme $Q:=\{q=0\} \subset \mathbf{P}_{R}^{n-1}$ is smooth over $R$. Let $u \in R^{\times}$be a unit. If $u$ is represented by $q$ over $K$, then $u$ is represented by $q$ already over $R$.

If $1 / 2 \in R$, then the same holds true for a quadratic space of an arbitrary rank.

Proof. The proof is literally the same as the proof of the same result in the case of characteristic not equal to 2 , given in $\mathrm{PaP}$, Corollary 3.3]. The even rank condition provides that in the course of the proof after adding a summand of rank 1 one obtains a semiregular quadratic form of odd rank.

\section{§2. Proof of Proposition 2}

Proof. Since the conclusion of the proposition only depends on the affine scheme $X$ locally in the Zariski topology, we may replace $X$ by its appropriate open affine subscheme containing $x$. The following lemma is a corollary to Lemma 2.2.1 and Proposition 3.1.7. in [Kn, Chapter IV].

Lemma 7. For $n>1$, there exists an affine open subset $X^{0}$ containing $x$ and a Galois étale cover $\overline{X^{0}} \stackrel{\rho}{\rightarrow} X^{0}$ such that the $k\left[X^{0}\right]$-module $P \otimes_{k[X]} k\left[X^{0}\right]$ is isomorphic to $k\left[X^{0}\right]^{n}$ 
and $\rho^{*}(q)$ is proportional to the quadratic form $\perp_{i=1}^{m} T_{i} T_{i+m}$ in the case of $n=2 m$ and is proportional to the semiregular quadratic form $\perp_{i=1}^{m} T_{i} T_{i+m} \perp T_{n}^{2}$ in the case of $n=2 m+1$.

Taking this lemma into account, we may assume the quadric $Q$ to be locally split for the étale topology.

Further, by [PSV, Propositions 2.3 and 2.4] one can find an open $X^{\prime}$ in $X$ containing $x$ and an open affine $S \subset \mathbf{P}^{d-1}(d=\operatorname{dim}(X))$ and a smooth morphism $r^{\prime}: X^{\prime} \rightarrow S$ turning $X^{\prime}$ into a smooth relative curve over $S$ with geometrically irreducible fibers. Moreover, the morphism $r^{\prime}$ could be chosen so that

- its restriction $\left.r^{\prime}\right|_{X^{\prime} \cap Z}: Z^{\prime}=X^{\prime} \cap Z \rightarrow S$ is a finite morphism, where $Z$ is the vanishing locus of $f \in k[X]$;

- it factors as the composition $X^{\prime} \stackrel{\Pi^{\prime}}{\longrightarrow} \mathbf{A}^{1} \times S \stackrel{\mathrm{pr}_{S}}{\longrightarrow} S$, where $\Pi^{\prime}: X^{\prime} \rightarrow \mathbf{A}^{1} \times S$ is a finite surjective morphism.

Below we write $X$ for $X^{\prime}, Z$ for $Z^{\prime}, r: X \rightarrow S$ for $r^{\prime}: X^{\prime} \rightarrow S$, and $\Pi_{S}: X \rightarrow \mathbf{A}^{1} \times S$ for $\Pi^{\prime}: X^{\prime} \rightarrow \mathbf{A}^{1} \times S$.

Denote by $\mathcal{X}=U \times_{S} X \stackrel{r_{U}}{\longrightarrow} U$ the base change of the morphism $r$ under the composition $U \stackrel{\text { can }}{\longrightarrow} X \stackrel{r}{\rightarrow} S$, where can: $U \rightarrow X$ is a canonical inclusion. Obviously, this morphism $r_{U}$ has a section $\Delta: U \rightarrow \mathcal{X}$ induced by can. Denote by $\Pi: \mathcal{X} \rightarrow \mathbf{A}^{1} \times U$ the base change of the morphism $\Pi_{S}$ under the map $U \rightarrow S$. Finally, denote by $f_{U} \in k[\mathcal{X}]$ the element $\operatorname{pr}_{X}^{*}(f)$, where $\operatorname{pr}_{X}: \mathcal{X}=U \times_{S} X \rightarrow X$ is the projection to the second factor.

One can easily check that $\left(r_{U}: \mathcal{X} \rightarrow U, f_{U}, \Delta\right)$ is a nice triple as defined in [PSV] Definition 3.1]. Actially, we merely recalled the construction of a basic nice triple from [PSV, $\S 6]$. Denote by ${ }_{U} \mathcal{Q}$ the quadric over $\mathcal{X}$ that is the pullback of the quadric ${ }_{U} Q$ under the morphism $r_{U}$. Denote by $\mathcal{Q}_{X}$ the quadric over $\mathcal{X}$ that is a pullback of a quadric $Q$ under the morphism $\operatorname{pr}_{X}: \mathcal{X} \rightarrow X$.

Proposition 8. There exist a finite étale covering $\theta: \tilde{\mathcal{X}} \rightarrow \mathcal{X}$ turning $\tilde{\mathcal{X}}$ into a relative curve with geometrically connected fibers over $U$ such that

- there is a section $\widetilde{\Delta}: U \rightarrow \widetilde{\mathcal{X}}$ with $\theta \circ \widetilde{\Delta}=\Delta$;

- there is an isomorphism of $\tilde{\mathcal{X}}$-schemes $\Phi: \theta^{*}\left(\mathcal{Q}_{X}\right) \rightarrow \theta^{*}\left({ }_{U} \mathcal{Q}\right)$;

- there is a finite surjective morphism $\pi: \widetilde{\mathcal{X}} \rightarrow \mathbf{A}^{1} \times U$ over $U$.

Proof. Since our $X$ has been chosen to be sufficiently small, by Lemma 7 an isomorphism class of a quadric $Q$ is attached to a certain cohomological class in $H_{e t}^{1}\left(X, \mathbf{P} \mathbf{G} \mathbf{O}_{n}\right)$. Therefore, the quadrics $\mathcal{Q}_{X}$ and ${ }_{U} \mathcal{Q}$ correspond to their classes $\alpha_{X}, \alpha_{U} \in H_{e t}^{1}\left(\mathcal{X}, \mathbf{P G O}_{n}\right)$. In order to find an isomorphism between two $\widetilde{\mathcal{X}}_{\text {-schemes }} \theta^{*}\left(\mathcal{Q}_{X}\right)$ and $\theta^{*}\left({ }_{U} \mathcal{Q}\right)$, it suffices to check that $\theta^{*}\left(\alpha_{X}\right)=\theta^{*}\left(\alpha_{U}\right)$ in $H_{e t}^{1}\left(\tilde{\mathcal{X}}, \mathbf{P G O} \mathbf{O}_{n}\right)$.

By [PSV, Theorem 3.6], for given classes $\alpha_{X}, \alpha_{U} \in H_{e t}^{1}\left(\mathcal{X}, \mathbf{P G O}_{n}\right)$ there exists a morphism of nice triples $(\widetilde{r}: \widetilde{\mathcal{X}} \rightarrow U, \tilde{f}, \widetilde{\Delta}) \stackrel{\theta}{\rightarrow}\left(r_{U}: \mathcal{X} \rightarrow U, f_{U}, \Delta\right)$ providing the required equality $\theta^{*}\left(\alpha_{X}\right)=\theta^{*}\left(\alpha_{U}\right)$.

Taking into account the conclusion of the last proposition, we are under the hypotheses of Lemma 10 from Appendix A for the relative $U$-curve $\tilde{\mathcal{X}}$ and its closed subset $\mathcal{Z}$ defined by an equation $f_{U} \circ \theta=0$. (More precisely, then one should take the connected component $\tilde{\mathcal{X}}^{c}$ of $\tilde{\mathcal{X}}$ containing $\widetilde{\Delta}(U)$ and the closed subset $\mathcal{Z} \cap \widetilde{\mathcal{X}}^{c}$ of $\tilde{\mathcal{X}}^{c}$.)

By Lemma 10, there exists an open subscheme $\mathcal{X}^{0} \hookrightarrow \tilde{\mathcal{X}}$ and a finite surjective morphism $\alpha: \mathcal{X}^{0} \rightarrow \mathbf{A}^{1} \times U$ such that $\alpha$ is étale over $0 \times U$ and $1 \times U$ and $\alpha^{-1}(0 \times U)=$ $\widetilde{\Delta}(U) \coprod D_{0}$. Moreover, if we define $D_{1}$ as $\alpha^{-1}(1 \times U)$, then $D_{1} \cap \mathcal{Z}=\varnothing$ and $D_{0} \cap \mathcal{Z}=\varnothing$. One has $\left[D_{1}: U\right]=\left[D_{0}: U\right]+1$. Thus, either $\left[D_{1}: U\right]$ is odd or $\left[D_{0}: U\right]$ is odd. 
Without loss of generality we assume that $\left[D_{1}: U\right]$ is odd, otherwise taking $D_{0}$ for $D_{1}$. Since $D_{1} \cap \mathcal{Z}=\varnothing$, the image of the composition $D_{1} \hookrightarrow \widetilde{\mathcal{X}} \stackrel{\theta}{\rightarrow} \mathcal{X} \stackrel{\mathrm{pr}_{X}}{\rightarrow} X$ does not intersect $Z$. Therefore, we obtaine the morphism $D_{1} \rightarrow X_{f}=X-Z$, the morphism $1 \times U \stackrel{\left.\alpha\right|_{D_{1}}}{\longleftarrow} D_{1}$ and the isomorphism $\bar{\Phi}:=\left.\Phi\right|_{D_{1}}$ satisfying the conclusion of Proposition 2 (here $\Phi$ is from Proposition 8). The proposition is proved.

Remark 9. At the end of the proof of Proposition 2. one can use the isomorpism $\Phi$ constructed in Proposition 11 instead of that from Proposition 8 . The former proposition does not rely on [PSV, Theorem 3.6] and has an elementary proof which is valid, however, only when the characteristic of the ground field $k$ is not equal to 2 .

\section{§3. Appendix A: A version of the Geometric lemma}

Let $k$ be an infinite field, $X$ a $k$-smooth algebraic variety, $x \in X$ a point, $\mathcal{O}=\mathcal{O}_{X, x}$ the local ring, and let $U=\operatorname{Spec}(\mathcal{O})$. Let $\mathcal{X} / U$ be a $U$-smooth relative curve with geometrically connected fibres equipped with a finite surjective morphism $\pi: \mathcal{X} \rightarrow \mathbf{A}^{1} \times U$ and with a section $\Delta: U \rightarrow \mathcal{X}$ of the projection $p: \mathcal{X} \rightarrow U$. Let $\mathcal{Z} \subset \mathcal{X}$ be a closed subset finite over $U$. The following lemma is a version of Lemma 5.1 in OP.

Lemma 10. There exists an open subscheme $\mathcal{X}^{0} \hookrightarrow \mathcal{X}$ and a finite surjective morphism $\alpha: \mathcal{X}^{0} \rightarrow \mathbf{A}^{1} \times U$ such that $\alpha$ is étale over $0 \times U$ and $1 \times U$ and $\alpha^{-1}(0 \times U)=\Delta(U) \coprod D_{0}$. Moreover, such $\mathcal{X}^{0}$ and $\alpha$ can be chosen so that if we define $D_{1}$ as $\alpha^{-1}(1 \times U)$, then $D_{1} \cap \mathcal{Z}=\varnothing$ and $D_{0} \cap \mathcal{Z}=\varnothing$.

Proof. Let $\overline{\mathcal{X}}$ be the normalization of the scheme $\mathbf{P}^{1} \times U$ in the function field $k(\mathcal{X})$ of $\mathcal{X}$. Let $\bar{\pi}: \overline{\mathcal{X}} \rightarrow \mathbf{P}^{1} \times U$ be the morphism. Let $\mathcal{X}_{\infty}=\bar{\pi}^{-1}(\infty \times U)$ be the set theoretic preimage of $\infty \times U$. Let $\bar{p}: \overline{\mathcal{X}} \rightarrow U$ be the structure map. Let $u \in U$ be the closed point, and let $\bar{X}_{u}=\overline{\mathcal{X}} \times_{U} u$.

Let $L^{\prime}=\bar{\pi}^{*}\left(\mathcal{O}_{\mathbf{P}^{1} \times U}(1)\right), L^{\prime \prime}=\mathcal{O}_{\overline{\mathcal{X}}}(\Delta(U))$. Let $D_{\infty}=\left(\bar{\pi}^{*}\right)(\infty \times U)$ be the pull-back of the Cartier divisor $\infty \times U \subset \mathbf{P}^{1} \times U$. Choose and fix a closed embedding $i: \overline{\mathcal{X}} \hookrightarrow \mathbf{P}^{n} \times U$ of $U$-schemes. Set $L=i^{*}\left(\mathcal{O}_{\mathbf{P}^{n} \times U}(1)\right)$.

The sheaf $L$ is very ample. Thus, the sheaf $L^{\prime \prime} \otimes L$ is also very ample. So, there exists a closed embedding $i^{\prime \prime}: \overline{\mathcal{X}} \hookrightarrow \mathbf{P}^{n^{\prime \prime}} \times U$ of $U$-schemes such that $L^{\prime \prime} \otimes L=\left(i^{\prime \prime}\right)^{*}\left(\mathcal{O}_{\mathbf{P}^{n^{\prime \prime}} \times U}(1)\right)$. Using the Bertini theorem, we can choose a hyperplane $H^{\prime \prime} \subset \mathbf{P}^{n^{\prime \prime}} \times U$ such that

$\left(a^{\prime \prime}\right) H^{\prime \prime} \cap \Delta(U)=\varnothing, H^{\prime \prime} \cap \mathcal{Z}=\varnothing, H^{\prime \prime} \cap D_{\infty}=\varnothing$.

Define a Cartier divisor $D^{\prime \prime}$ on $\overline{\mathcal{X}}$ as the closed subscheme $H^{\prime \prime} \cap \overline{\mathcal{X}}$ of $\overline{\mathcal{X}}$. Regard $D_{1}^{\prime \prime}:=D^{\prime \prime} \coprod D_{\infty}$ as a Cartier divisor on $\overline{\mathcal{X}}$. Clearly, $\mathcal{O}_{\overline{\mathcal{X}}}\left(D_{1}^{\prime \prime}\right)=L^{\prime \prime} \otimes L \otimes L^{\prime}$.

The sheaf $L$ is very ample. Thus, the sheaf $L^{\prime} \otimes L$ is also very ample. So, there exists a closed embedding $i^{\prime}: \overline{\mathcal{X}} \hookrightarrow \mathbf{P}^{n^{\prime}} \times U$ of $U$-schemes such that $L^{\prime} \otimes L=\left(i^{\prime}\right)^{*}\left(\mathcal{O}_{\mathbf{P}^{n^{\prime}} \times U}(1)\right)$. Using the Bertini theorem, we can choose a hyperplane $H^{\prime} \subset \mathbf{P}^{n^{\prime}} \times U$ such that

$\left(a^{\prime}\right) H^{\prime} \cap \Delta(U)=\varnothing, H^{\prime} \cap \mathcal{Z}=\varnothing, H^{\prime} \cap D_{1}^{\prime \prime}=\varnothing ;$

$\left(b^{\prime}\right)$ the scheme theoretic intersection $H^{\prime} \cap \bar{X}_{u}$ is a $k(u)$-smooth scheme.

Define a Cartier divisor $D^{\prime}$ on $\overline{\mathcal{X}}$ as the closed subscheme $D^{\prime}=H^{\prime} \cap \overline{\mathcal{X}}$ of $\overline{\mathcal{X}}$.

Regard $D_{1}^{\prime}:=D^{\prime} \coprod \Delta(U)$ as a Cartier divisor on $\overline{\mathcal{X}}$. Clearly, $\mathcal{O}_{\overline{\mathcal{X}}}\left(D_{1}^{\prime}\right)=L^{\prime} \otimes L \otimes L^{\prime \prime}$.

Observe that $D^{\prime}$ is an essentially $k$-smooth scheme finite and étale over $U$. Let $s^{\prime}$ and $s^{\prime \prime}$ be global sections of $L^{\prime} \otimes L \otimes L^{\prime \prime}$ such that the vanishing locus of $s^{\prime}$ is the Cartier divisor $D_{1}^{\prime}$ and the vanishing locus of $s^{\prime \prime}$ is the Cartier divisor $D_{1}^{\prime \prime}$. Clearly, $D_{1}^{\prime} \cap D_{1}^{\prime \prime}=\varnothing$. Thus, the morphism $f=\left[s^{\prime}: s^{\prime \prime}\right]: \overline{\mathcal{X}} \rightarrow \mathbf{P}^{1}$ is regular elsewhere. Set

$$
\bar{\alpha}=(f, \bar{p}): \overline{\mathcal{X}} \rightarrow \mathbf{P}^{1} \times U .
$$

Clearly, $\bar{\alpha}$ is a finite surjective morphism. Set $\mathcal{X}^{0}=\bar{\alpha}^{-1}\left(\mathbf{A}^{1} \times U\right)$ and

$$
\alpha=\left.\bar{\alpha}\right|_{\mathcal{X}^{0}}: \mathcal{X}^{0} \rightarrow \mathbf{A}^{1} \times U \text {. }
$$


Clearly, $\alpha$ is a finite surjective morphism and $\mathcal{X}^{0}$ is an open subscheme of $\mathcal{X}$. Since $\alpha$ is a finite surjective morphism and $\mathcal{X}^{0}$ and $\mathbf{A}^{1} \times U$ are regular schemes, the morphism $\alpha$ is flat by a theorem of Grothendieck. Since $D_{1}^{\prime}$ is finite étale over $U$, the morphism $\alpha$ is étale over $0 \times U$. So, we may choose a point $1 \in \mathbf{P}^{1}$ such that $\alpha$ is étale over $1 \times U$ and $(\alpha)^{-1}(1 \times U) \cap \mathcal{Z}=\varnothing$. If we set $D_{0}=D_{1}^{\prime}$, then $\alpha^{-1}(0 \times U)=\Delta(U) \amalg D_{0}$ and $D_{0} \cap \mathcal{Z}=\varnothing$. The lemma is proved.

\section{$\S 4$. Appendix B}

In this section we give an "elementary" proof of a version of Proposition 8 that is valid in characteristic not equal to 2 but does not require a reference to a general equating theorem, see [PSV], Theorem 3.6].

In the case of characteristic not equal to 2, a regular quadratic space is diagonalizable locally in the Zariski topology. Therefore, we may assume that

$$
q\left(T_{1} e_{1}+\cdots+T_{n} e_{n}\right)=a_{1} T_{1}^{2}+\cdots+a_{n} T_{n}^{2},
$$

where $a_{i} \in k[X]^{*}$. Consider two quadratic spaces $q_{1}$ and $q_{2}$ over $\mathcal{X}=X \times_{S} X$ induced by the quadratic space $q$ over $X$ by projections on the first and the second factor, respectively.

Proposition 11. There exist an étale covering $\theta: \tilde{\mathcal{X}} \rightarrow \mathcal{X}$ such that

- quadratic spaces $\theta^{*} q_{1}$ and $\theta^{*} q_{2}$ over $\tilde{\mathcal{X}}$ are isomorphic;

- there is a morphism $\widetilde{\Delta}: X \rightarrow \tilde{\mathcal{X}}$ such that the composition $\theta \circ \widetilde{\Delta}$ coincides with the diagonal embedding $\Delta: X \rightarrow X \times{ }_{S} X=\mathcal{X}$.

Proof. We take $\tilde{\mathcal{X}}$ to be an affine scheme such that its coordinate ring is equal to $k[X] \otimes_{k[S]} k[X]\left[\sqrt{a_{1}} \otimes \sqrt{a_{1}}, \ldots, \sqrt{a_{n}} \otimes \sqrt{a_{n}}\right]$. Since $a_{i} \in k[X]^{*}$, it follows that $k[\widetilde{\mathcal{X}}]$ is an étale extension of $k[\mathcal{X}]=k[X] \otimes_{k[S]} k[X]$. The quadratic space $\theta^{*}\left(q_{1}\right)$ is written as $\perp_{i=1}^{n}\left(a_{i} \otimes 1\right) T_{i}^{2}$ in the chosen coordinates, and the quadratic space $\theta^{*}\left(q_{2}\right)$ is written as $\perp_{i=1}^{n}\left(1 \otimes a_{i}\right) T_{i}^{2}$. Since $\left(a_{i} \otimes 1\right) \cdot\left(1 \otimes a_{i}\right)$ is a square in $k[\tilde{\mathcal{X}}]$, these quadratic spaces are isomorphic. Consider a fiber of the morphism $\theta$ over $\Delta(X)$ :

$$
k[\Delta] \otimes_{k[\tilde{\mathcal{X}}]}\left(k[X] \otimes_{k[S]} k[X]\right)=k[X]\left[t_{1}, \ldots, t_{n}\right] /\left(t_{1}^{2}-a_{1}^{2}, \ldots, t_{1}^{2}-a_{n}^{2}\right)=\bigoplus_{r=1}^{2^{n}} k[X] .
$$

Choosing a summand, one constructs a suitable section $\widetilde{\Delta}$.

\section{REFERENCES}

[EKM] R. Elman, N. Karpenko, and A. Merkurjev, The algebraic and geometric theory of quadratic forms, Amer. Math. Soc. Colloquium Publ., vol. 56, Amer. Math. Soc., Providence, RI, 2008. $\operatorname{MR} 2427530$

[Kn] M. A. Knus, Quadratic and hermitian forms over rings, Grundlehren Math. Wiss., Bd. 294, Springer-Verlag, Berlin, 1991. MR1096299

[OP] M. Ojanguren and I. A. Panin, A purity theorem for the Witt group, Ann. Sci. Ecole Norm. Sup. (4) 32 (1999), no. 1, 71-86. MR1670591

[PR] I. A. Panin and U. Rehmann, A variant of a theorem by Springer, Algebra i Analiz 19 (2007), no. 6, 117-125; English transl., St. Petersburg Math. J. 19 (2008), no. 6, 953-959; www. math.uiuc.edu/K-theory/0671/2003 MR2411641

[Pa] I. A. Panin, Rationally isotropic quadratic spaces are locally isotropic, Invent. Math. 176 (2009), no. 2, 397-403. MR2495767

[PaP] I. A. Panin and K. I. Pimenov, Rationally isotropic quadratic spaces are locally isotropic. II, Doc. Math. 2010, Extra Vol.: Andrei A. Suslin's sixtieth birthday, 515-523. MR2804263

[PSV] I. A. Panin, A. K. Stavrova and N. A. Vavilov, On Grothendieck-Serre's conjecture concerning principal G-bundles over reductive group schemes. I, Compos. Math. 151 (2015), no. 3, 535-567. $\operatorname{MR} 3320571$ 
St. Petersburg Branch, Steklov Mathematical Institute, Russian Academy of Sciences, Fontanka 27, 191023 St. Petersburg, Russia

E-mail address: panin@pdmi.ras.ru

Department of Mathematics and Mechanics, St. Petersburg State University, UniverSitetskil Pr. 28, Petergof, 198504 St. Petersburg, Russia

E-mail address: kip302002@yahoo.com

Received 15/JUN/2015

Originally published in English 